\title{
ORDERS OF TORSION UNITS OF INTEGRAL REALITY-BASED ALGEBRAS WITH RATIONAL MULTIPLICITIES
}

\author{
GURMAIL SINGH AND ALLEN HERMAN*
}

\begin{abstract}
A reality-based algebra (RBA) is a finite-dimensional associative algebra with involution over $\mathbb{C}$ whose distinguished basis $\mathbf{B}$ contains 1 and is closed under pseudo-inverse. An integral $\mathrm{RBA}$ is one whose structure constants in its distinguished basis are integers. If the algebra has a one-dimensional representation taking positive values on $\mathbf{B}$, then we say that the RBA has a positive degree map. These RBAs have a standard feasible trace, and the multiplicities of the irreducible characters in the standard feasible trace are the multiplicities of the RBA. In this article we show that for integral RBAs with positive degree map whose multiplicities are rational, any finite subgroup of torsion units whose elements are all of degree 1 and have algebraic integer coefficients must have order dividing a certain positive integer determined by the degree map and the multiplicities. The paper concludes with a thorough investigation of the properties of RBAs that force multiplicities to be rational.
\end{abstract}

\section{INTRODUCTION}

Reality-based algebras (RBAs) were introduced by Blau in [4] to unify treatments of various types of hypergroups that have been discussed in recent literature. Group algebras of finite groups, finitedimensional Hecke algebras, Brauer's pseudogroups, Bose-Mesner algebras of finite association schemes (see [19]), the fusion algebras and $\mathbb{Z}$-based algebras arising in studies of integral modular data (see [8]), table algebras (see [1]), doubly Frobenius algebras, and Kawada's $C$ algebras are all special types of reality-based algebras.

It is natural to ask which familiar properties of groups or group rings hold in the general setting of reality-based algebras? The authors have recently extended several basic results concerning torsion units of

2000 Mathematics Subject Classification. Primary 16U60; Secondary 05E30, 16S99, 20N99.

Key words and phrases. Reality based algebras, table algebras, fusion algebras, group of units, torsion units, multiplicities.

*The work of this author has been supported by an NSERC Discovery Grant. 
integral group rings to torsion units of $\mathbb{Z} \mathbf{B}$ where $\mathbf{B}$ is a standard basis of an integral RBA with standard character; i.e. in the situation where the multiplicity of every irreducible character of $\mathbb{C B}$ in the standard feasible trace of $\mathbb{C B}$ is a positive integer (see [12], [13], [16], and [11]). After reviewing background terminology and properties for RBAs in Section 2, in Section 3 we adapt the approach of [13] to the situation where the multiplicities are rational. We show that the order of any torsion unit of $\mathbb{Z} \mathbf{B}$ is a divisor of a positive integer determined explicitly by the values of the degree map on $\mathbf{B}$ and the multiplicities of the RBA. In Section 4, we investigate the general question of when an RBA with positive degree map has rational multiplicities, and give several sufficient critieria.

\section{ReAlity-BASED ALGEBRAS}

A reality based algebra (RBA) is a pair $(A, \mathbf{B})$, where $A$ is a finitedimensional algebra over $\mathbb{C}$ with an $\mathbb{R}$-linear and $\mathbb{C}$-conjugate linear involution $*: A \rightarrow A$, and $\mathbf{B}=\left\{b_{0}, b_{1}, \ldots, b_{d}\right\}$ is a distinguished basis of $A$ that satisfies the following properties:

(i) $b_{0}=b_{0}^{*}=1_{A} \in \mathbf{B}$;

(ii) there is a transposition $*$ of $\{1, \ldots, d\}$ such that $\left(b_{i}\right)^{*}=b_{i^{*}}$, for all $i \in\{1, \ldots, d\}$,

(iii) the structure constants of $A$ with respect to the basis $\mathbf{B}$ are real numbers, i.e. for all $b_{i}, b_{j} \in \mathbf{B}$, we have

$$
b_{i} b_{j}=\sum_{b_{k} \in \mathbf{B}} \lambda_{i j k} b_{k}, \text { for some } \lambda_{i j k} \in \mathbb{R},
$$

(iv) for all $b_{i}, b_{j} \in \mathbf{B}, \lambda_{i j 0} \neq 0 \Longleftrightarrow j=i^{*}$,

(v) for all $b_{i} \in \mathbf{B}, \lambda_{i i^{*} 0}=\lambda_{i^{*} i 0}>0$.

If these conditions are satisfied, we say that $\mathbf{B}$ is an RBA-basis. The algebra $A$ is a $C^{*}$-algebra whose involution satisfies $\alpha^{*}=\sum_{i} \bar{\alpha}_{i} b_{i^{*}}$ when $\alpha=\sum_{i} \alpha_{i} b_{i} \in A$ is written as a $\mathbb{C}$-linear combination of the RBAbasis. In particular, $A$ is a $(d+1)$-dimensional semisimple algebra. Being a finite-dimensional $C^{*}$-algebra, any $\operatorname{RBA}(A, \mathbf{B})$ has a faithful *-representation, and in fact any simple component of $A$ has a faithful *-representation (see [17, Theorem 11.2]).

An $\operatorname{RBA}(A, \mathbf{B})$ has a positive degree map if there is a $*$-algebra homomorphism $\delta: A \rightarrow \mathbb{C}$ for which $\delta\left(b_{i}\right)=\delta\left(b_{i^{*}}\right)>0$ for all $b_{i} \in \mathbf{B}$. If $(A, \mathbf{B})$ is an RBA with positive degree map $\delta$, then we say that its RBA-basis $\mathbf{B}$ is standard when $\delta\left(b_{i}\right)=\lambda_{i i^{*} 0}$, for all $b_{i} \in \mathbf{B}$. Every RBA with positive degree map has a unique standard basis, which can 
be arranged by rescaling each basis element by $\delta\left(b_{i}\right) / \lambda_{i i^{*} 0}$. When the $\operatorname{RBA}(A, \mathbf{B})$ admits a positive degree map $\delta$, we define its order to be

$$
n:=\delta\left(\mathbf{B}^{+}\right)=\sum_{i=0}^{d} \delta\left(b_{i}\right)
$$

and its standard feasible trace to be $\rho: A \rightarrow \mathbb{C}$ with $\rho(\alpha)=n \alpha_{0}$ for all $\alpha=\sum_{i} \alpha_{i} b_{i} \in A$. Note that $\rho$ really is a trace function on $A$ because $\rho(\alpha \beta)=\rho(\beta \alpha)$ for all $\alpha, \beta \in A$. Positivity of the degree map means that $A$ can be made into a Frobenius algebra, with nondegenerate hermitian form given by

$$
\langle\alpha, \beta\rangle=\rho\left(\alpha \beta^{*}\right), \quad \text { for all } \alpha, \beta \in A .
$$

Being a nonsingular trace function on the finite-dimensional Frobenius algebra $A, \rho$ can be expressed as a linear combination of the irreducible characters of $A$ [14]. The coefficients $m_{\chi}$ in this linear combination $\rho=\sum_{\chi} m_{\chi} \chi$ are the multiplicities of an RBA with positive degree map. Higman's character formula [14]

$$
e_{\chi}=\frac{m_{\chi}}{n} \sum_{i} \frac{\chi\left(b_{i}^{*}\right)}{\delta\left(b_{i}\right)} b_{i}
$$

expresses the centrally primitive idempotents of $A$ in terms of the standard basis $\mathbf{B}$. Since $\chi\left(b_{i^{*}}\right)=\overline{\chi\left(b_{i}\right)}$ for all $b_{i} \in \mathbf{B}$, we have

$$
\chi\left(e_{\chi}\right)=\chi\left(b_{0}\right)=\frac{m_{\chi}}{n} \sum_{i} \frac{\overline{\chi\left(b_{i}\right)} \chi\left(b_{i}\right)}{\delta\left(b_{i}\right)} .
$$

$\chi\left(b_{0}\right)$ is the degree of $\chi$ so it is always a positive integer. When the degrees $\delta\left(b_{i}\right)$ are all positive and real, then $n$ is positive and real, so the multipicity $m_{\chi}$ is positive and real.

If $(A, \mathbf{B})$ is an RBA and $R$ is a subring of $\mathbb{C}$ that contains all the structure constants relative to the basis $\mathbf{B}$, then the $R$-span of the basis $\mathbf{B}$ is an $R$-free $R$-subalgebra of $A$ of rank $d+1$, which we will denote by $R \mathbf{B}$. When $(A, \mathbf{B})$ is an RBA with positive degree map and the structure constants relative to the standard basis $\mathbf{B}$ are integers (resp. rationals), then we say that $(A, \mathbf{B})$ is an integral (resp. rational) $R B A$ with positive degree map. For an integral RBA with positive degree map, the ring $\mathbb{Z} \mathbf{B}$ is reminiscent of the integral group ring of a finite group. Motivated by what is known about units of integral group rings of finite groups, we ask: what are the possible orders of torsion units of $\mathbb{Z} \mathbf{B}$ when $\mathbf{B}$ is the standard basis of an integral RBA? 


\section{TORSION UNITS OF INTEGRAL RBAS WITH RATIONAL MULTIPLICITIES}

Suppose $(A, \mathbf{B})$ is an integral RBA with positive degree map for which the multiplicities are rational. (This is the same as the "standard character" condition discussed by Blau in [4, Section 2].) Let $N$ be the least positive integer for which $N \rho=\sum_{\chi}\left(N m_{\chi}\right) \chi$ is a nonnegative integer linear combination of the irreducible characters of $A$. Since every irreducible representation of $A$ is realized by a $*$-representation, there is a $*$-representation $\Gamma$ that affords the character $N \rho$. Since $\Gamma\left(b_{0}\right)=N \rho\left(b_{0}\right)=N n$, the degree of $\Gamma$ is $N n$, where $n$ is the order of the RBA.

Lemma 1. Let $(A, \mathbf{B})$ be an $R B A$ with positive degree map that has rational multiplicities. Let $\rho$ be the standard feasible trace of $A$, and let $N$ be the least positive integer such that $N \rho$ is a character of $A$. Suppose $\Gamma$ is a *-representation of $A$ affording $N \rho$. Then $\left\{\Gamma\left(b_{i}\right): b_{i} \in \mathbf{B}\right\}$ is a linearly independent set.

Proof. Suppose $\sum_{0}^{d} \alpha_{j} \Gamma\left(b_{j}\right)=0$ where $\alpha_{j} \in \mathbb{C}$. Let $i \in\{0,1, \ldots, d\}$. Multiplying this equation by $\Gamma\left(b_{i}^{*}\right)$ on both sides and taking the trace gives $0=\sum_{j} \alpha_{j} N \rho\left(b_{j} b_{i}^{*}\right)=\alpha_{i} \lambda_{i i^{*} 0} N n$, so $\alpha_{i}=0$. Therefore, $\left\{\Gamma\left(b_{i}\right)\right.$ : $\left.b_{i} \in \mathbf{B}\right\}$ is a linearly independent set.

The above lemma is also a consequence of the fact that the multiplicities of the RBA are all positive, since this implies that there are no nonzero two-sided ideals in the kernel of $\Gamma$.

Now we turn our attention to torsion units. We begin with a lemma that is analogous to the Berman-Higman lemma for torsion units of integral group rings.

Lemma 2. Let $(A, \mathbf{B})$ be a rational $R B A$ with positive degree map that has rational multiplicities. Suppose $u=\sum_{i} u_{i} b_{i}$ is a torsion unit of $A$ whose coefficients $\left\{u_{i}\right\}$ in the standardized $R B A$-basis $\mathbf{B}$ are algebraic integers. If $u_{0} \neq 0$, then $u=\delta(u) b_{0}$.

Proof. Let $\rho$ be the standard feasible trace of $(A, \mathbf{B})$, and let $N$ be the least positive integer for which $N \rho$ is a character of $A$. Suppose the multiplicative order of $u$ is $k$. Let $\Gamma$ be a $*$-representation of $A$ affording $N \rho$. By Lemma $1, \Gamma(u)$ is a matrix of finite order $k$, and so is a diagonalizable matrix whose eigenvalues are $k$-th roots of unity. Let $\zeta_{k}^{f_{1}}, \ldots, \zeta_{k}^{f_{N n}}$ be the eigenvalues of $\Gamma(u)$, where $\zeta_{k}$ is a fixed primitive $k$ th root of unity in $\mathbb{C}$. Then $N n u_{0}=N \rho(u)=\sum_{i=1}^{N n} \zeta_{k}^{f_{i}}$. Taking absolute 
values we get

$$
N n\left|u_{0}\right|=\left|\sum_{i=1}^{N n} \zeta_{k}^{f_{i}}\right| \leq N n .
$$

Therefore, $\left|u_{0}\right| \leq 1$, and when equality holds, all of the $\zeta_{k}^{f_{i}}$ are equal to $u_{0}$.

Now let $\sigma \in \operatorname{Gal}(\overline{\mathbb{Q}} / \mathbb{Q})$, with $\overline{\mathbb{Q}}$ being an algebraic closure of $\mathbb{Q}$. Since the structure constants in the basis B are rational, $\sigma$ induces a $\mathbb{Q}$ algebra automorphism of $\overline{\mathbb{Q}} \mathbf{B}$ that fixes $\mathbf{B}$ and acts by $\sigma$ on coefficients. In particular, $u^{\sigma}$ is another torsion unit of $A$ with algebraic integer coefficients. By the above reasoning, it follows that $\left|\left(u^{\sigma}\right)_{0}\right|=\left|u_{0}^{\sigma}\right| \leq 1$. Therefore, $u_{0}$ is an algebraic integer whose Galois conjugates all have norm at most 1, so by a classical result of Kroenecker we can conclude that either $u_{0}$ is 0 or a root of unity. Since $u_{0} \neq 0$, it follows that $\left|u_{0}\right|=1$, so equality holds in (1).

This implies that $\Gamma(u)=u_{0} I=u_{0} \Gamma\left(b_{0}\right)$, so by Lemma $1, u=u_{0} b_{0}$.

Proposition 3. Let $(A, \mathbf{B})$ be an $R B A$ with positive degree map and rational multiplicities. Let $\rho$ be the standard feasible trace of $A$, and let $N$ be the least positive integer such that $N \rho$ is a character of $A$. Let $e=\sum_{i} e_{i} b_{i}$ be a non-zero idempotent of $A=\mathbb{C B}$.

Then $N n e_{0} \in \mathbb{Z}^{+}$and $1 \leq N n e_{0} \leq N n$. Furthermore, $e_{0}=1$ precisely when $e=b_{0}$.

Proof. Let $\Gamma$ be a $*$-representation of $A$ affording the character $N \rho$. If $e=\sum_{i} e_{i} b_{i} \in \mathbb{C} \mathbf{B}$ is a non-zero idempotent, then

$$
\operatorname{rank}(\Gamma(e))=\operatorname{tr}(\Gamma(e))=N \rho(e)=\sum_{i} e_{i} N \rho\left(b_{i}\right)=e_{0} N n .
$$

Since $\operatorname{rank}(\Gamma(e))$ is a positive integer satisfying $1 \leq \operatorname{rank}(\Gamma(e)) \leq N n$, the result follows.

Remark 4. In the special case where $e_{\chi}$ is one of the centrally primitive idempotents of $A$, and $\Gamma$ is a*-representation affording the character $N \rho$, then because the $e_{\chi}$ for $\chi \in \operatorname{Irr}(A)$ are orthogonal and sum to $1_{A}=b_{0}$, it must be the case that the rank of $\Gamma\left(e_{\chi}\right)$ is $N m_{\chi} \chi\left(b_{0}\right)$ for every $\chi \in \operatorname{Irr}(A)$.

If $(A, \mathbf{B})$ is an RBA with positive degree map, we will say that a unit $u$ of $A$ is normalized when $\delta(u)=1$.

Lemma 5. Let $(A, \mathbf{B})$ be a rational $R B A$ with positive degree map and rational multiplicities. Then any finite group of normalized torsion 
units having algebraic integer coefficients in the standardized RBA-basis $\mathbf{B}$ is a set of $\mathbb{C}$-linearly independent elements of $A$.

Proof. Let $T$ be a finite group of normalized units of $A$ whose elements have algebraic integer coefficients. Suppose $T$ is not linearly independent. Let $\alpha_{1} t_{1}+\ldots+\alpha_{m} t_{m}=0$ be an expression of minimal positive length $m$, where the $t_{i}$ are elements of $T$ and the coefficients $\alpha_{1}, \ldots, \alpha_{m} \in \mathbb{C}$ are all nonzero. Since $T$ is a group, we can assume without loss of generality that $t_{1}=b_{0}$. Since $T$ consists of normalized torsion units we have by Lemma 2 that $\left(t_{j}\right)_{0}=0$ for $j=2, \ldots, m$. It follows that

$$
0=\left(\alpha_{1} b_{0}+\alpha_{2} t_{2}+\cdots+\alpha_{m} t_{m}\right)_{0}=\alpha_{1},
$$

a contradiction. Therefore, $T$ must be a linearly independent set.

The main result of [13] gives a Lagrange theorem for finite subgroups of normalized units of integral RBAs with standard character, which says the order of the subgroup divides the order of the RBA. We now establish a version of this theorem for rational RBAs with positive degree map and rational multiplicities.

Theorem 6. Let $(A, \mathbf{B})$ be a rational $R B A$ with positive degree map and rational multiplicities. Suppose $(A, \mathbf{B})$ has order $n$ and rank $r$. Let $\rho$ be the standard feasible trace of $(A, \mathbf{B})$, and let $N$ be the least positive integer such that $N \rho$ is a character of $A$.

Let $T$ be a finite subgroup of normalized units having algebraic integer coefficients in the standardized RBA-basis $\mathbf{B}$. Then the order of $T$ divides $N n$ and is at most $r$.

Proof. By Lemma 5, $T$ is a linearly independent subset of $A$. Since $A$ has dimension $r,|T| \leq r$.

Let $e=\frac{1}{|T|} \sum_{t \in T} t$. Since $T$ is a finite group, $e$ is a nonzero idempotent of $A$. Let $\Gamma$ be a *-representation of $A$ affording $N \rho$, and let $m=\operatorname{rank}(\Gamma(e))=N \rho(e)$. Note that, being the rank of a nonzero idempotent, $m$ is a positive integer.

Also $m=N \rho(e)=\frac{1}{|T|} \sum_{t \in T} N \rho(t)=\frac{1}{|T|} \sum_{t \in T} N n t_{0}$. If $t$ is an element of $T$ other than $b_{0}$, then Lemma 2 implies $t_{0}=0$. Therefore, $m=N \rho(e)=\frac{N n}{|T|}$, and we have that $|T|$ divides $N n$.

\section{Conditions for RBAs to have Rational multiplicities}

Let $(A, \mathbf{B})$ be an RBA with positive degree map, and suppose $\mathbf{B}$ is a standard basis. In this section we will determine conditions for the multiplicities of $(A, \mathbf{B})$ to be rational. 
In general, if the structure constants relative to the standard basis B lie in a field $F$, then let $K$ be the algebraic extension of $F$ obtained by adjoining entries of the character table of $(A, \mathbf{B})$. We will refer to $K$ as the field of realization of the character table of $A$. (This should not be confused with the splitting field of the RBA over $F$, which could be a larger field when the RBA is not commutative.) It is easy to see that $\operatorname{Gal}(K / F)$ permutes the irreducible characters of $A$, since for all $\chi \in \operatorname{Irr}(A)$, the fact that $\sigma \in \operatorname{Gal}(K / F)$ implies that $\chi^{\sigma}$ is the character of a Galois conjugate representation of $A$. Let $H$ be the subset of $\operatorname{Gal}(K / F)$ whose elements are realized by column permutations of the character table, that is, $\sigma \in H$ if and only if there is a permuation $\bar{\sigma}$ of $\mathbf{B}$ such that for all $\chi \in \operatorname{Irr}(A)$, and for all $b_{i} \in \mathbf{B}$, $\chi\left(b_{i}\right)^{\sigma}=\chi\left(b_{i}^{\bar{\sigma}}\right)$. It is easy to see that the identity of $\operatorname{Gal}(K / F)$ lies in $H$, and that $H$ is a subgroup of $\operatorname{Gal}(K / F)$. In fact, $H$ is a central subgroup of $\operatorname{Gal}(K / F)$, because

$$
\chi\left(b_{i}\right)^{\tau \sigma}=\left(\chi^{\tau}\left(b_{i}\right)\right)^{\sigma}=\chi^{\tau}\left(b_{i}^{\bar{\sigma}}\right)=\left(\chi\left(b_{i}^{\bar{\sigma}}\right)\right)^{\tau}=\chi\left(b_{i}\right)^{\sigma \tau}
$$

for all $\sigma \in H$ and $\tau \in \operatorname{Gal}(K / F)$. The fact that $\overline{\chi\left(b_{i}\right)}=\chi\left(b_{i}^{*}\right)$ holds for characters of RBAs implies that complex conjugation is always realized by an element of $H$.

In the special case when the $\operatorname{RBA}(A, \mathbf{B})$ with positive degree map $(A, \mathbf{B})$ is commutative, it is called a $C$-algebra. The character table $P$ of a $C$-algebra is its first eigenmatrix, and the unique matrix $Q$ for which $P Q=n I$ is its second eigenmatrix. Kawada-Delsarte duality tells us the second eigenmatrix is the character table of the dual $C$-algebra $(\hat{A}, \hat{\mathbf{B}})$ in its standard basis [2, Theorem II.5.10]. The multiplicities of $(A, \mathbf{B})$ are precisely the degrees of the dual, which are listed in the first row of $Q=n P^{-1}$. A commutative $C$-algebra $(A, \mathbf{B})$ is self-dual when we can permute the rows and columns of $P$ to obtain a $C$-algebra isomorphism between $A$ and its dual. As complex conjugation is realized by a column permutation of the character table, this is equivalent to the existence of permutation matrices $U$ and $V$ for which $U Q V=\bar{P}$.

We now gather some easy conditions that imply rational multiplicities.

Theorem 7. Let $(A, \mathbf{B})$ be a rational $R B A$ with positive degree map $\delta$ and standard basis $\mathbf{B}$. Then any of the following conditions forces all multiplicities of $(A, \mathbf{B})$ to be rational.

(i) The standard feasible trace of $(A, \mathbf{B})$ is a character of $A$.

(ii) $(A, \mathbf{B})$ has a rational character table.

(iii) For every $\chi \in \operatorname{Irr}(A), \mathbb{Q}$ is the real subfield of $\mathbb{Q}(\chi)$. 
(iv) All Galois automorphisms acting on elements of the character table are realized by column permutations of the character table.

(v) $(A, \mathbf{B})$ has only two multiplicities, 1 and $t$.

(vi) $(A, \mathbf{B})$ is commutative and self-dual.

(vii) $(A, \mathbf{B})$ is commutative with order $n$, and its first eigenmatrix $P$ has the property that the first row of $P^{2}$ is $(n, 0, \ldots, 0)$.

Proof. (i). The standard feasible trace $\rho=\sum_{\chi} m_{\chi} \chi$ is a character precisely when all of the multiplicities $m_{\chi}$ are positive integers.

(ii) and (iii). Suppose $\chi \in \operatorname{Irr}(A)$. If $n$ is the order of $(A, \mathbf{B})$, then applying $\chi$ to the centrally primitive idempotent

$$
e_{\chi}=\frac{m_{\chi}}{n} \sum_{i=0}^{d} \frac{\chi\left(b_{i^{*}}\right)}{\delta\left(b_{i}\right)} b_{i}
$$

gives

$$
\chi\left(b_{0}\right)=\chi\left(e_{\chi}\right)=\frac{m_{\chi}}{n} \sum_{i=0}^{d} \frac{\left|\chi\left(b_{i}\right)\right|^{2}}{\delta\left(b_{i}\right)} .
$$

Since $(A, \mathbf{B})$ is rational, all the degrees $\delta\left(b_{i}\right)$ for $b_{i} \in \mathbf{B}$ are rational, and $n$ and $\chi\left(b_{0}\right)$ are rational. Since $\overline{\chi\left(b_{i}\right)}=\chi\left(b_{i}^{*}\right),\left|\chi\left(b_{i}\right)\right|^{2}$ lies in $\mathbf{Q}(\chi)^{+}$, the real subfield of $\mathbf{Q}(\chi)$. When the $\left|\chi\left(b_{i}\right)\right|^{2}$ are rational for all $b_{i}$, equation (2) tells us $m_{\chi}$ is rational. This proves (ii) and (iii).

(iv). Since the standard basis $\mathbf{B}$ has rational structure constants, the values of irreducible characters of $A$ are algebraic over $\mathbb{Q}$. Let $K$ be the field of realization of the character table of $A$, and let $H$ be the central subgroup of $\operatorname{Gal}(K / F)$ whose elements are realized by column permutations of the character table. If $\sigma \in H$, then it will fix $\sum_{i}\left|\chi\left(b_{i}\right)\right|^{2}$. Since the $\delta\left(b_{i}\right), n$, and $\chi\left(b_{0}\right)$ all lie in $\mathbb{Q}$, by applying $\sigma$ to both sides of equation (2) we see that $\sigma$ must also fix $m_{\chi}$. This proves (iv).

(v). (This simple argument is due to the referee.) Let $\phi$ be the sum of the irreducible characters of $A$ with multiplicity 1 , and $\theta$ the sum of the irreducible characters with multiplicity $t$. Then $n=\rho\left(b_{0}\right)=$ $\phi\left(b_{0}\right)+t \theta\left(b_{0}\right)$ implies that $t$ is rational.

(vi). When $(A, \mathbf{B})$ is self-dual, we can initially re-order the columns of $P$ and columns of $Q$ so that $Q=\bar{P}$. The multiplicities of $A$ are precisely the degrees of the dual. The first row of $P$ gives the list of degrees of $A$, and the first row of $Q$, which corresponds to the degree map on $\hat{A}$, gives the list of multiplicities of $A$. Both the first row of $Q$ and the first row of $P$ are real, so when $Q=\bar{P}$, it means the list of 
multiplicities is an exact match for the list of degrees. When $(A, \mathbf{B})$ is rational and self-dual, the degrees are rational. This proves (vi).

(vii). Since $Q P=n I$ and the first row of $Q$ is the list of multplicities $\mathbf{m}$, we always have that $\mathbf{m} P=(n, 0, \ldots, 0)$. The first row of $P$ is the list of degrees $\mathbf{d}$, so the first row of $P^{2}$ is $\mathbf{d} P$. Since $P$ is invertible, $\mathbf{d} P=\mathbf{m} P$ implies $\mathbf{d}=\mathbf{m}$. This proves (vii).

Remark 8. The reasoning in (iii) shows the multiplicities of the RBA lie in the fixed field $K^{H}$. In the commutative case, the column permutations of the first eigenmatrix $P$ are in one-to-one correspondence with row permutations of the second eigenmatrix, so the structure constants of $(\hat{A}, \hat{\mathbf{B}})$ will lie in $K^{H}$. These structure constants are the Krein parameters of $(A, \mathbf{B})$. (That the Krein parameters are fixed by $H$ has been noted previously in [2] and [5, (2.14)].)

Condition (vii) is equivalent in general for the list of multiplicities to match the list of degrees for a $C$-algebra. The non-symmetric commutative association scheme as8-14 of [3] with order 8, rank 6, and degrees $1,1,1,1,2$, and 2 gives a non-self dual example in which the first row of $P^{2}$ satisfies condition (vii).

An important class of commutative table algebras that satisfies condition (vi) arises in studies of modular data. A $(d+1) \times(d+1)$ unitary and symmetric matrix $S$ is a Fourier matrix when every $S_{i 0}>0$ and $S$ generates integer structure constants using the Verlinde's formula:

$$
N_{i j k}=\sum_{\ell} S_{\ell 0}^{-1} S_{\ell i} S_{\ell j} \bar{S}_{\ell k}, 0 \leq i, j, k \leq d .
$$

These are the structure constants of the fusion ring associated with $S$. Conformal field theorists think of this as the fusion ring generated by the column vectors of the $s$-matrix under ordinary addition and entrywise multiplication, where $s$ is obtained from $S$ by dividing each row of $S$ by its first entry $S_{i 0}$. It turns out that this fusion ring is the transitional rescaling of a self-dual commutative table algebra. The Fourier matrix $S$ occurs in a modular datum when there exists a diagonal torsion matrix $T$ for which $S^{2}=(S T)^{3}$. This means $S$ and $T$ are the images of the usual generators of the modular group $S L_{2}(\mathbb{Z})$ under a unitary finite-dimensional group representation. Readers interested in connections with the subject of rational conformal field theory are referred to [8].

Corollary 9. The following families of RBAs with degree map have rational multiplicities:

(i) Adjacency algebras of finite association schemes, 
(ii) Commutative or noncommutative t-pseudocyclic rational RBAs, and

(iii) Commutative rational table algebras whose transitional rescaling is the fusion algebra of an integral modular datum.

(iv) Self-dual standard C-algebras with square degrees whose transitional rescaling has rational structure constants.

Proof. (i). Adjacency algebras of association schemes are RBAs whose standard feasible trace is a character.

(ii). A $t$-pseudocyclic RBA is by definition an RBA with positive degree map that has only two multiplicities, 1 and $t$.

(iii). Let $(A, \mathbf{B})$ be a commutative standard table algebra whose transitional rescaling $(A, \tilde{\mathbf{B}})$ is isomorphic as a table algebra to a fusion ring arising from an integral modular datum. Then $\tilde{\mathbf{B}}$ has integral structure constants $N_{i j k}$, and the standard table algebra $(A, \mathbf{B})$ is selfdual. However, the structure constants $\lambda_{i j k}$ relative to the basis $\mathbf{B}$ are not necessarily rational, since $\lambda_{i j k}=\frac{\sqrt{\delta\left(b_{i}\right) \delta\left(b_{j}\right)}}{\sqrt{\delta\left(b_{k}\right)}} N_{i j k}$, for all $i, j, k$. When these structure constants are rational, Theorem 7(vi) applies.

(iv) Reasoning as in the proof of (iii) above, $(A, \mathbf{B})$ will be a commutative rational self-dual RBA, so it has rational multiplicities by Theorem 7 (vi).

For RBAs with rational-valued positive degree map, it follows from the proof of Theorem 7 that the multiplicity of any rational-valued irreducible character is rational. In particular, if the character table is rational, then the multiplicities are rational. This is easily seen to include all rational RBAs of rank 2 and the noncommutative rational RBAs of rank 5 or 6 discussed in [9] and [10]. We will give a small example to show the multiplicities of a rank 3 RBA can be irrational.

Example 10. Let $\mathbf{B}=\left\{b_{0}=1, b_{1}, b_{2}\right\}$ be the standard basis of a symmetric integral table algebra whose structure constants are given by

$$
b_{1}^{2}=3 b_{0}+2 b_{2}, \quad b_{1} b_{2}=2 b_{1}+b_{2}, \text { and } b_{2}^{2}=3 b_{0}+b_{1}+b_{2} .
$$

Since this algebra is commutative, we can produce the character table for $(A, \mathbf{B})$ by diagonalizing the regular matrices for $b_{1}$ and $b_{2}$. Here it is, with $\sigma$ denoting the nontrivial element of $\operatorname{Gal}(\mathbb{Q}(\sqrt{2}) / \mathbb{Q})$ :

\begin{tabular}{c|ccc|c} 
& $b_{0}$ & $b_{1}$ & $b_{2}$ & mult. \\
\hline$\delta$ & 1 & 3 & 3 & 1 \\
$\chi$ & 1 & $-1+\sqrt{2}$ & $-\sqrt{2}$ & $m_{\chi}$ \\
$\chi^{\sigma}$ & 1 & $-1-\sqrt{2}$ & $\sqrt{2}$ & $\left(m_{\chi}\right)^{\sigma}$
\end{tabular}


Calculating directly using the above formulas, we find $m_{\chi}=3+\frac{3}{4} \sqrt{2}$.

While this example does not have the rational multiplicity condition we need for our results in the previous section, since it is commutative we know by [18, Theorem 3.1] that the only torsion units of $\mathbb{Z} \mathbf{B}$ are $\pm b_{0}$.

Example 10 is a 3 -homogeneous $P$-polynomial integral table algebra, corresponding to the intersection array $[3,2 ; 1,2]$. An RBA is $k$ homogeneous if every degree $\delta\left(b_{i}\right)$ is a fixed positive number $k$ except for $\delta\left(b_{0}\right)=1$. Multiplicities of $k$-homogeneous $P$-polynomial integral table algebras were investigated recently by Blau and Hein [6], who described infinite families having rank 6 or more with irrational multiplicities.

Remark 11. Another aspect of the $C$-algebra theory described in [2] is a method for calculating structure constants from the character table. If one starts with the character table $P$ of a $C$-algebra with basis $\mathbf{B}$, and calculates structure constants for the algebra spanned by the column vectors of $P$ under entrywise multiplication, one recovers regular matrices for the elements of $\mathbf{B}$. For instance the reader can check that when this is applied to the character table $P$ of Example 10, one obtains the regular matrices of the standard basis elements for this RBA.

Example 12. The integral table algebra of rank 3 in Example 10 has irrational multiplicities, so it also has irrational Krein parameters. From the character table $Q$ of the dual $\hat{A}$, we can calculate the Krein parameters of $(A, \mathbf{B})$ directly using Remark 11 .

$$
Q=7 P^{-1}=\left[\begin{array}{ccc}
1 & 3+\frac{3}{4} \sqrt{2} & 3-\frac{3}{4} \sqrt{2} \\
1 & -\frac{1}{2}+\frac{3}{4} \sqrt{2} & -\frac{1}{2}-\frac{3}{4} \sqrt{2} \\
1 & -\frac{1}{2}-\sqrt{2} & -\frac{1}{2}+\sqrt{2}
\end{array}\right]
$$

Taking the columns of $Q$ as basis vectors for an algebra with entrywise product, and writing the regular matrices corresponding to each, we obtain

$b_{0}^{\prime}=I_{3}, b_{1}^{\prime}=\left[\begin{array}{ccc}0 & 3+\frac{3}{4} \sqrt{2} & 0 \\ 1 & \frac{3}{4}+\frac{5}{8} \sqrt{2} & \frac{5}{4}+\frac{1}{8} \sqrt{2} \\ 0 & \frac{7}{4}+\frac{7}{8} \sqrt{2} & \frac{5}{4}-\frac{1}{8} \sqrt{2}\end{array}\right], b_{2}^{\prime}=\left[\begin{array}{ccc}0 & 0 & 3-\frac{3}{4} \sqrt{2} \\ 0 & \frac{5}{4}+\frac{1}{8} \sqrt{2} & \frac{7}{4}-\frac{7}{8} \sqrt{2} \\ 1 & \frac{5}{4}-\frac{1}{8} \sqrt{2} & \frac{3}{4}-\frac{5}{8} \sqrt{2}\end{array}\right]$.

The entries are irrational, which is to be expected because the Galois automorphism of $\operatorname{Gal}(\mathbb{Q}(\sqrt{2}) / \mathbb{Q})$ is not realized by a row permutation of $Q$. So although $(A, \mathbf{B})$ is an integral $C$-algebra, its dual is neither 
rational nor integral, and the structure constants relative to the standard basis of the dual are not algebraic integers. In this case they are not positive either - note that the $(3,3)$ entry of $b_{2}^{\prime}$ is negative.

For commutative association schemes, the argument showing the Krein parameters are nonnegative relies on two key points. First, the centrally primitive idempotents are realized as nonnegative definite Hermitian matrices in the standard $*$-representation affording $\rho$, and second, the multiplication for the dual algebra is realized by entrywise matrix multiplication in the standard representation (see $[2$, $\S I I .3])$. When the multiplicities are rational, the centrally primitive idempotents will be realized by nonnegative definite matrices by our *-representation affording our $N \rho$, but it may not be the case that the multiplication in the dual algebra corresponds to entrywise matrix multiplication for this representation.

Example 13. The rank 4 table algebra whose standard basis $\mathbf{B}=$ $\left\{b_{0}, b_{1}, b_{2}, b_{3}\right\}$ satisfies

$$
\begin{array}{ll}
b_{1}^{2}=3 b_{0}+b_{1}+b_{2}, & b_{2}^{2}=3 b_{0}+2 b_{2}, \\
b_{1} b_{2}=b_{1}+2 b_{2}, & b_{2} b_{3}=2 b_{1}+b_{3}, \\
b_{1} b_{3}=2 b_{2}+b_{3}, & b_{3}^{2}=3 b_{0}+b_{1}+b_{2}
\end{array}
$$

has character table

$$
P=\left[\begin{array}{cccc}
1 & 3 & 3 & 3 \\
1 & -2 & 3 & -2 \\
1 & 2 & -1 & -2 \\
1 & -1 & -1 & 1
\end{array}\right]
$$

Its multiplicities are $1, \frac{3}{2}, \frac{5}{2}$, and 5 , so it will have a degree 20 representation affording $2 \rho$. Two of its Krein parameters, in particular $\kappa_{122}=\kappa_{212}=-\frac{1}{6}$ and $\kappa_{221}=-\frac{5}{18}$, are negative. Therefore, multiplication for the dual algebra cannot correspond to entrywise matrix multiplication in this representation.

It was shown by Hosseini and Rahnamai Barghi that for integral $k$-homogeneous table algebras of rank 3 , rationality of multiplicities implies rationality of Krein parameters [15, Theorem 3.2]. We remark that their proof can be applied without change to $k$-homogeneous rank $3 C$-algebras. It is quite common for $C$-algebras with rational multiplicities to have irrational Krein parameters. The $P$-polynomial table algebra corresponding to the Heawood graph, with incidence array 
$[3,2,2 ; 1,1,3]$, is one such example. Its eigenmatrices are

$$
P=\left[\begin{array}{cccc}
1 & 3 & 6 & 4 \\
1 & -3 & 6 & -4 \\
1 & \sqrt{2} & -1 & -\sqrt{2} \\
1 & -\sqrt{2} & -1 & \sqrt{2}
\end{array}\right] \text { and } Q=\left[\begin{array}{cccc}
1 & 1 & 6 & 6 \\
1 & -1 & 2 \sqrt{2} & -2 \sqrt{2} \\
1 & 1 & -1 & -1 \\
1 & -1 & -\frac{3}{2} \sqrt{2} & \frac{3}{2} \sqrt{2}
\end{array}\right]
$$

and one can show $\kappa_{333}=\frac{5}{2}+\frac{1}{4} \sqrt{2}$.

The authors would like to express their appreciation to the referee for their helpful comments and suggestions for this article.

\section{REFERENCES}

[1] Z. Arad, E. Fisman, and M. Muzychuk, Generalized table algebras, Israel J. Math., 114 (1999), 29-60.

[2] E. Bannai and T. Ito, Algebraic Combinatorics I: Association Schemes, Benjamin/Cummings, Menlo Park, CA, 1984.

[3] A. Hanaki and I. Miyamoto, Classification of Small Association Schemes, (http://math.shinshu-u.ac.jp/ ${ }^{\sim}$ hanaki/as/)

[4] H. I. Blau, Table algebras, European J. Combin., 30 (2009), no. 6, 1426-1455.

[5] H. I. Blau, Quotient structures in C-algebras, J. Algebra, 175 (1995), no. 1, 24-64; Erratum: 177 (1995), no. 1, 297-337.

[6] H. I. Blau and R. J. Hein, A class of $P$-polynomial table algebras with and without integer multiplicities, Comm. Algebra, 42 (12), (2014), 5387-5424.

[7] M. Cuntz, Integral modular data and congruences, J. Algebraic Comb., 29, (2009), 357-387.

[8] T. Gannon, Modular data: the algebraic combinatorics of conformal field theory, J. Algebraic Comb., 22, (2005), 211-250.

[9] A. Herman, M. Muzychuk, and B. Xu, The recognition problem for table algebras and reality-based algebras, preprint, arXiv:1506.05476v2 [math.RA].

[10] A. Herman, M. Muzychuk, and B. Xu, Noncommutative reality-based algebras of rank 6, preprint, arXiv:1608.08463v1 [math.RA].

[11] A. Herman and G. Singh, Central torsion units of integral reality-based algebras with positive degree map, Int. Electron. J. Algebra, 21 (2017), 121-126.

[12] A. Herman and G. Singh, On the Torsion Units of Integral Adjacency Algebras of Finite Association Schemes, Algebra, Vol. 2014, 2014, Article ID 842378, 5 pages.

[13] A. Herman and G. Singh, Torsion units of integral C-algebras, JP J. Algebra Number Theory Appl., 36 (2), 2015, 141-155.

[14] D. G. Higman, Coherent algebras, Linear Algebra Appl., 93 (1987), 209-239.

[15] A. Hosseini and A. Rahnamai Barghi, Table algebras of rank 3 and its applications to strongly regular graphs, J. Algebra Appl., 12 (5) (2013), 125-141.

[16] G. Singh, Torsion Units of Integral Group Rings and Scheme Rings, Ph.D. Thesis, University of Regina, 2015.

[17] M. Takesaki, Theory of Operator Algebras I, Encyclopaedia of Mathematical Sciences, Vol. 124, Springer, Berlin, 1979. 
[18] B. Xu, On Isomorphisms Between Integral Table Algebras and Applications to Finite Groups and Association Schemes, Comm. Algebra, 42 (2014), no. 12, 5249-5263.

[19] P.-H. Zieschang, Theory of Association Schemes, Springer Monographs in Mathematics, Springer-Verlag, Berlin, 2005.

Department of Mathematics and Statistics, University of Regina, Regina, Canada, S4S 0A2

E-mail address: Gurmail.Singh@uregina.ca

Department of Mathematics and Statistics, University of Regina, Regina, Canada, S4S 0A2

E-mail address: Allen.Herman@uregina.ca 